\title{
Molecular photoacoustic imaging of breast cancer using an actively targeted conjugated polymer
}

\author{
This article was published in the following Dove Press journal: \\ International Journal of Nanomedicine \\ 8 January 2015 \\ Number of times this article has been viewed
}

\author{
Ghayathri Balasundaram ${ }^{1, *}$ \\ Chris Jun Hui Hol,* \\ Kai $\mathrm{Li}^{2}$ \\ Wouter Driessen ${ }^{3}$ \\ US Dinish' \\ Chi Lok Wong' \\ Vasilis Ntziachristos ${ }^{3}$ \\ Bin Liu ${ }^{2}$ \\ Malini Olivo ${ }^{1,4}$ \\ 'Bio-Optical Imaging Group, \\ Singapore Bioimaging Consortium \\ (SBIC), ${ }^{2}$ Institute of Materials \\ Research and Engineering (IMRE), \\ Agency for Science, Technology \\ and Research (A*STAR), Singapore; \\ ${ }^{3}$ Institute of Biological and Medical \\ Imaging, Helmholtz Center Munich, \\ Neuherberg, Germany; ${ }^{4}$ School \\ of Physics, National University \\ of Ireland, Galway, Ireland \\ *These authors contributed equally \\ to this work
}

\begin{abstract}
Conjugated polymers (CPs) are upcoming optical contrast agents in view of their unique optical properties and versatile synthetic chemistry. Biofunctionalization of these polymer-based nanoparticles enables molecular imaging of biological processes. In this work, we propose the concept of using a biofunctionalized CP for noninvasive photoacoustic (PA) molecular imaging of breast cancer. In particular, after verifying the PA activity of a CP nanoparticle ( $\mathrm{CP}$ dots) in phantoms and the targeting efficacy of a folate-functionalized version of the same (folate-CP dots) in vitro, we systemically administered the probe into a folate receptorpositive (FR+ve) MCF-7 breast cancer xenograft model to demonstrate the possible application of folate-CP dots for imaging FR+ve breast cancers in comparison to $\mathrm{CP}$ dots with no folate moieties. We observed a strong PA signal at the tumor site of folate-CP dots-administered mice as early as 1 hour after administration as a result of the active targeting of the folate-CP dots to the FR+ve tumor cells but a weak PA signal at the tumor site of CP-dots-administered mice as a result of the passive accumulation of the probe by enhanced permeability and retention effect. We also observed that folate-CP dots produced $\sim 4$-fold enhancement in the PA signal in the tumor, when compared to $\mathrm{CP}$ dots. These observations demonstrate the great potential of this active-targeting $\mathrm{CP}$ to be used as a contrast agent for molecular PA diagnostic imaging in various biomedical applications.
\end{abstract}

Keywords: photoacoustic tomography, conjugated polymers, molecular imaging, breast cancer

\section{Introduction}

Photoacoustic (PA) imaging is an emerging imaging technique based on the concept of laser-generated ultrasound, combining the high absorption contrast and specificity of optical imaging with the high spatial resolution and penetration depth of ultrasound imaging. ${ }^{1-3}$ To generate PA signals, an ultrafast laser pulse irradiates the tissue, and absorption of the light energy causes the tissue to undergo a transient thermoelastic expansion that produces acoustic waves. This imaging method is versatile, being able to provide multifaceted information: anatomical imaging based on endogenous tissue absorption, functional imaging of blood oxygenation, as well as molecular imaging based on absorption of exogenous contrast agents. Thus, it has been widely used in various applications, including imaging of the brain, ${ }^{4-6}$ cancer, ${ }^{7,8}$ cardiovasculature,,${ }^{9,10}$ as well as the evaluation of gene delivery efficiency. ${ }^{11}$

In general, PA imaging can be performed using endogenous contrast based on tissue chromophores, such as oxy- and deoxyhemoglobin, or exogenous contrast agents for improved contrast and higher molecular sensitivity. In addition, for in vivo imaging, it is typically conducted in the near-infrared (NIR) region from 650 to $900 \mathrm{~nm}$, as there is low tissue absorption in this region, making it suitable for deep tissue imaging. Currently, various exogenous contrast agents have been explored for PA imaging, in 
view of their high intrinsic absorption properties in the NIR region. These include gold ${ }^{12-14}$ and silver ${ }^{15}$ nanostructures, single-walled carbon nanotubes, ${ }^{16}$ and NIR dyes such as indocyanine green. ${ }^{17-19}$ Although these compounds have shown a certain level of efficacy for imaging purposes, they have some inherent drawbacks, such as complex chemical procedures for tuning of optical properties of gold and silver nanostructures, water insolubility issues of pristine carbon nanotubes, as well as fast body clearance of small dyes, making them unsuitable for longitudinal studies.

On the other hand, conjugated polymers (CPs) are gaining significant attention in recent years as imaging contrast agents owing to their unique chemical and optical properties. ${ }^{20}$ These polymers are macromolecules with $\pi$-conjugated backbones, which enhance photoluminescence. In particular, the optoelectronic properties due to the highly delocalized $\pi$-electrons enable delicate tuning of the optical properties via backbone or side-chain modification. These polymers can be specially designed to display strong fluorescence for bioimaging purposes, which have been extensively used for in vitro cellular imaging ${ }^{21-23}$ and in vivo animal imaging. ${ }^{24}$ In recent years, researchers are starting to realize their potential as PA contrast agents. ${ }^{25,26}$ In this study, we employed a CP, poly[9,9-bis(4-(2-ethylhexyl)phenyl)fluorene-alt-co6,7-bis(4-(hexyloxy)phenyl)-4,9-di(thiophen-2-yl)thiadiazoloquinoxaline] (PFTTQ, Figure 1A), as the PA agent. Water-insoluble CP was made into water-dispersible nanoparticles (CP dots), functionalized with folate ligands (folate-CP

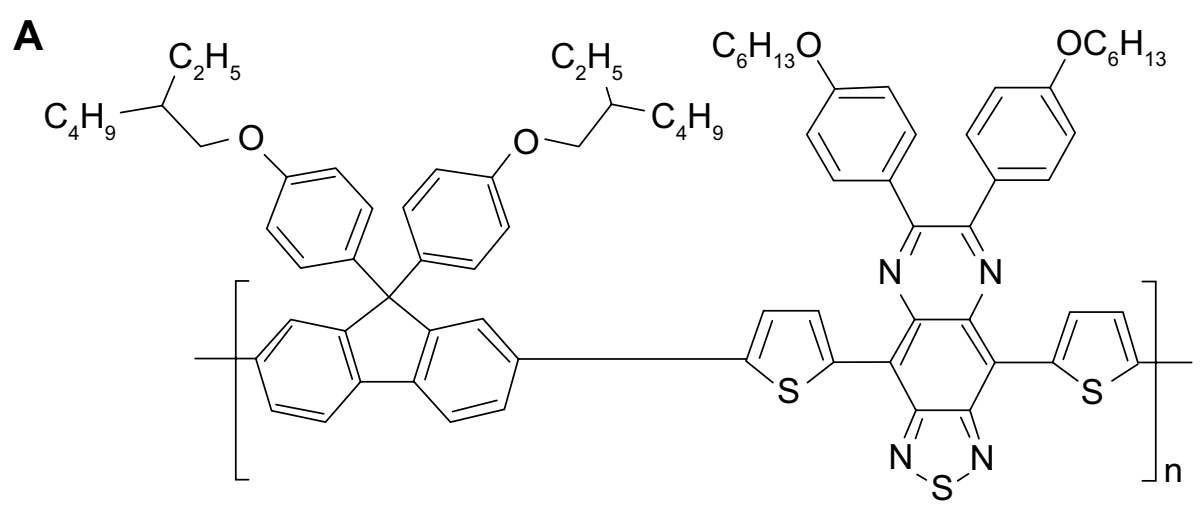

B

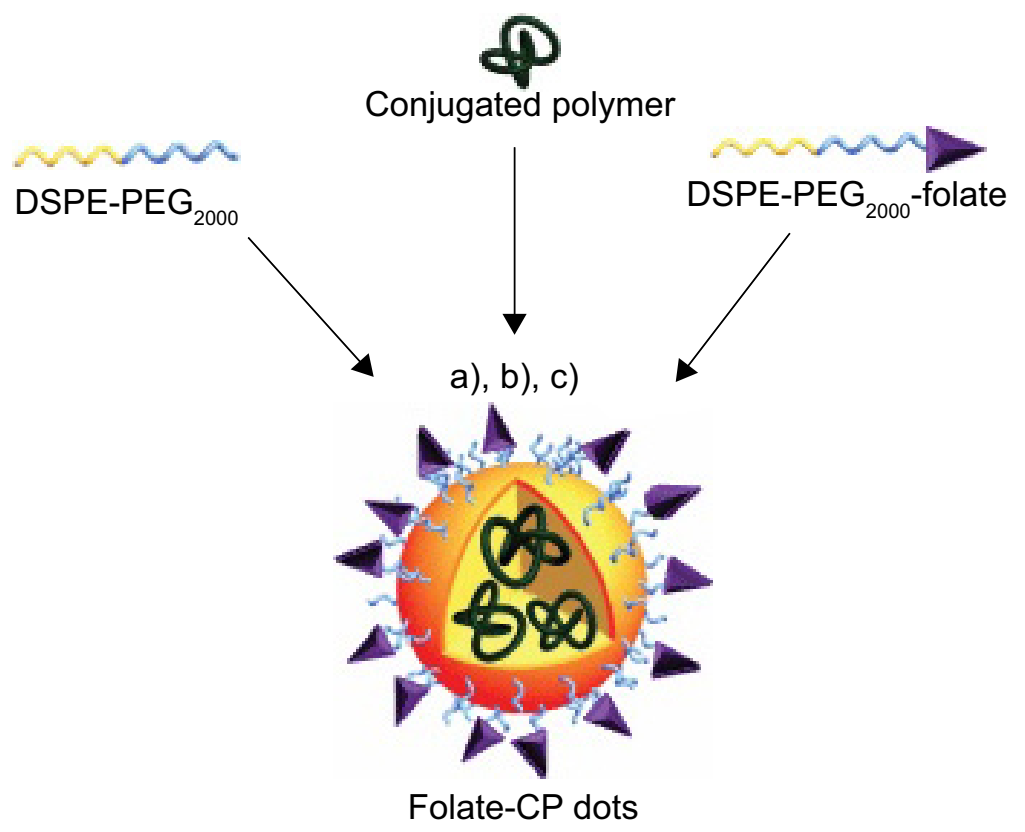

Figure I Preparation of folate ligand-functionalized conjugated polymers.

Notes: (A) Chemical structure of PFTTQ. (B) Schematic illustration of fabrication of folate-CP dots. Folate-CP dots were prepared by (a) addition of THF solution of PFTTQ, DSPE-PEG 2000 , and DSPE-PEG 2000 -folate into water; (b) sonification of the mixture for 2 minutes, and (c) THF evaporation under vigorous stirring overnight. Abbreviations: CP, conjugated polymer; DSPE-PEG ${ }_{2000}$, I,2-distearoyl-sn-glycero-3-phosphoethanolamine-N-[methoxy(polyethyleneglycol)-2000]; PFTTQ, poly[9,9-bis(4-(2ethylhexyl)phenyl)fluorene-alt-co-6,7-bis(4-(hexyloxy)phenyl)-4,9-di(thiophen-2-yl)thiadiazoloquinoxaline]; THF, tetrahydrofuran. 
dots), and employed for noninvasive molecular imaging of breast cancer using a multispectral optoacoustic tomography (MSOT) system. Upon systemic administration into a folate receptor-positive (FR+ve) breast cancer xenograft model, we observed strong PA signal at the tumor site of folate-CP dots-administered mice as early as 1 hour postadministration as a result of the active targeting of the folate-CP dots to the FR+ve tumor cells but a weak PA signal at the tumor site of $\mathrm{CP}$ dots-administered-administered mice as a result of the passive accumulation of the probe by enhanced permeability and retention (EPR) effect. We also observed that folate-CP dots produced a $\sim 4$-fold enhancement in the PA signal in the tumor, when compared to CP dots. These observations demonstrate a great potential of this active targeting $\mathrm{CP}$ to be used as a contrast agent for molecular PA diagnostic imaging in various biomedical applications.

\section{Material and methods Synthesis of folate-CP dots}

A tetrahydrofuran solution $(1 \mathrm{~mL})$ containing CP, DSPE-PEG ${ }_{2000}$ (1,2-distearoyl-sn-glycero-3-phosphoethanolamine- $N$ [methoxy(polyethyleneglycol)-2000]), and DSPE-PEG ${ }_{2000}{ }^{-}$ folate $(1 \mathrm{mg}$ each) was mixed with water $(10 \mathrm{~mL})$, followed by sonicating the mixture for 60 seconds at $12 \mathrm{~W}$ output using a microtip probe sonicator (XL2000, Misonix Inc., Farmingdale, NY, USA). The mixture was then stirred at room temperature overnight to evaporate the tetrahydrofuran to yield the final folate-CP dots suspended in water.

\section{Cell culture and in vitro assays}

MCF-7 breast cancer cells were cultured in Dulbecco's Modified Eagle's Medium (DMEM) containing 10\% fetal bovine serum and $1 \%$ penicillin streptomycin at $37^{\circ} \mathrm{C}$ in a humidified environment containing 5\% $\mathrm{CO}_{2}$. When $80 \%$ confluent, cells were used for cytotoxicity and specificity testing. For cytotoxicity studies, $1 \times 10^{4}$ cells were seeded per well in a 96-well plate and exposed to $0.025,0.05,0.075$, and $0.1 \mathrm{mg} / \mathrm{mL}$ of CP dots and folate-CP dots for 24 hours. Cell viability was measured using Cell Counting Kit-8 (CCK-8, Sigma-Aldrich, St Louis, MO, USA). For testing the targeting specificity, $3 \times 10^{5}$ cells were seeded per well in a 6 -well plate and exposed to $\mathrm{CP}$ dots and folate-CP dots $(0.03 \mathrm{mg} / \mathrm{mL}$ each) for 2 hours in the presence of various concentrations of free folic acid $(0,50$, and $100 \mu \mathrm{M})$. Treated cells were trypsinized, harvested, resuspended in phosphate-buffered saline $(200 \mu \mathrm{L})$, and loaded into the phantom to measure the PA signal from the treated cells against the cells with no treatment.

\section{UV absorbance measurement}

The wavelength-dependent absorption spectrum of the contrast agent was measured from 680 to $950 \mathrm{~nm}$ using a spectrophotometer (DU 730, Beckman Coulter, Placentia, CA, USA), which in turn was fed into MSOT system for multispectral postprocessing of PA signals.

\section{MSOT experimental parameters and protocol}

All phantom and in vivo mouse imaging experiments were performed using a real-time MSOT imaging system that has been described previously. ${ }^{27}$ Optical excitation was provided by an optical parametric oscillator with a tunable NIR wavelength range from 680 to $980 \mathrm{~nm}$, which was in turn pumped by a Q-switched Nd:YAG laser with a pulse duration of $10 \mathrm{~ns}$ and repetition rate of $10 \mathrm{~Hz}$. Light was delivered by a fiber bundle divided into 10 output arms to illuminate the sample from multiple angles around the imaging plane. PA signals were acquired using a 128-element concave transducer array spanning a circular arc of $270^{\circ}$. This transducer array has a central frequency of $5 \mathrm{MHz}$, which provided a transverse spatial resolution in the range of 150-200 $\mu \mathrm{m}$. One transverse image slice was acquired from each laser pulse, resulting in a frame rate of $10 \mathrm{~Hz}$. During image acquisition, the sample is translated through the transducer array along its axis across the volume region of interest (ROI), in order to capture the corresponding transverse image slices. Ultrasound gel is applied on the mouse skin surface for in vivo imaging, and measurements were recorded in temperature-controlled water medium for good acoustic coupling. An animal holder with a thin polyethylene membrane is used to prevent direct contact between the mouse and the water.

\section{Evaluation of PA activity of contrast agent in phantom model}

The phantom is made of polyurethane and is cylindrical in shape with a diameter of $2 \mathrm{~cm}$, which is specially designed to mimic the shape, size, and optical properties of the mouse. In addition, it has two inner cylindrical channels, each with an inner diameter of $3 \mathrm{~mm}$, one for holding the control medium and the other for holding the dissolved contrast agent in the same medium. The control medium and contrast agent suspension $(170 \mu \mathrm{L}$ each) were pipetted into the respective channels before inserting the phantom into a phantom holder, which in turn was placed in the MSOT imaging chamber to be imaged. For data acquisition, we set up a ROI of multiple transverse slices with a step size of $1 \mathrm{~mm}$ across the channel portion which contains the probe and control, applied excitation wavelengths 
from 680 to $900 \mathrm{~nm}$ with an interval of $10 \mathrm{~nm}$ for each transverse slice, and recorded the averaged PA signals from 20 frames for each wavelength and position.

\section{In vivo longitudinal monitoring of probe biodistribution in mouse xenograft model}

All animal experiments were performed in compliance with guidelines set by the Institutional Animal Care and Use Committee, Agency for Science, Technology and Research (A*STAR). Xenograft models were established on Balb/C nude mice by injecting subcutaneously into the right flank of mice a cell suspension $(0.2 \mathrm{~mL})$ containing $5 \times 10^{6} \mathrm{MCF}-7$ breast cancer cells and matrigel (BD Biosciences, San Jose, CA, USA) in 1:1 volume ratio. When the tumor volume reached a palpable size, $200 \mu \mathrm{L}$ of $0.3 \mathrm{mg} / \mathrm{mL}$ of each probe was injected intravenously through the tail vein of each mouse under $2 \%$ isoflurane in order to achieve final blood concentrations of approximately $0.03 \mathrm{mg} / \mathrm{mL}$. Five laser excitation wavelengths $(700,750,810,850$, and $900 \mathrm{~nm})$ were chosen for multispectral imaging on the basis of the absorption maxima and minima of the contrast agent, and oxy- and deoxyhemoglobin. For data acquisition, a ROI of multiple transverse slices was set up with a step size of $0.3 \mathrm{~mm}$ from the liver to the lower abdomen region, and the averaged PA signals from 20 frames for each wavelength and position were recorded. Multispectral imaging was performed prior to injection, and 1 hour, 3 hours, 5 hours, 1 day, 2 days, 3 days, and 1 week postinjection.

\section{Statistical analysis}

Statistical analysis was performed using Prism software (Graphpad, San Diego, CA, USA). Differences in uptake of the CPs were tested for significance using the unpaired $t$-test. $P$-values less than 0.1 were considered significant. Data were represented as mean \pm standard error of the mean.

\section{Image reconstruction and multispectral processing}

Images were reconstructed using a model-based approach ${ }^{28}$ for offline analysis. They were then corrected using a wavelength-dependent exponential decay model, assuming a uniform distribution of absorption and scattering properties, as well as constant tissue oxygenation. This is to compensate for the attenuation of excitation light in deep tissues. After image reconstruction, multispectral processing was performed to resolve individual components from the various chromophores - the contrast agent, and oxy- and deoxyhemoglobin in vivo. For each pixel in the image, the method fits the total measured optoacoustic spectrum to the known absorption spectra of the individual chromophores, on the basis of least-squares linear regression.

\section{Results and discussion Preparation of folate ligand-functionalized conjugated polymers}

$\mathrm{CP}$ dots functionalized with folate ligands on the surface were synthesized through a modified nanoprecipitation method, as illustrated in Figure 1B. ${ }^{26,29-30}$ A conjugated polymer, PFTTQ, was employed as the PA agent. The mixture of DSPE-PEG ${ }_{2000}$ and its folic acid end-capped derivative (DSPE-PEG ${ }_{2000}$-folate) was used to encapsulate PFTTQ to afford folate-CP dots. For instance, tetrahydrofuran solution containing PFTTQ, DSPE-PEG ${ }_{2000}$, and DSPE-PEG ${ }_{2000}$-folate (each $1 \mathrm{mg}$ ) was rapidly mixed with excess water under sonication. During this process, the hydrophobic lipid segments tend to embed in the aggregated hydrophobic PFTTQ core, while the hydrophilic PEG chains extend into the aqueous phase to render the dots with antifouling properties as well as abundant surface folic acid groups. Upon solvent evaporation, the water suspension of folate-CP dots was filtered through a $0.2 \mu \mathrm{m}$ syringe-driven filter for future use. In addition, the obtained folate-CP dots suspension showed excellent colloidal stability as no obvious precipitation could be observed after being stored at $4^{\circ} \mathrm{C}$ for several months, due to the presence of surface PEG segments.

\section{Characterization of folate-CP/CP dots}

Folate-CP dots prepared by nanoprecipitation method were characterized for size by laser light scattering. Their average hydrodynamic diameter was $\sim 51.5 \mathrm{~nm}$ with a narrow size distribution in water (Figure 2A). The folate-CP dots were spherical in shape and monodisperse in size, as observed from high-resolution transmission electron microscopy (Figure 2B). The dark dots can be attributed to the high electron density of PFTTQ encapsulated in the core. On the basis of the dot concentration, the average folic acid density on each folate-CP dot surface was determined to be $\sim 4,516$. The detailed calculation is described in the Supplementary materials.

Figure $2 \mathrm{C}$ shows the UV-vis absorption spectrum of folate-CP dots in water suspension (red line). The broad absorption spectrum from 700 to $850 \mathrm{~nm}$ with a peak at $\sim 810 \mathrm{~nm}$ makes folate-CP dots highly suitable for in vivo PA imaging. In addition, its absorbance spectrum differs significantly from those of the major background tissue chromophores such as oxy- and deoxyhemoglobin (Figure S1), facilitating accurate multispectral unmixing of individual PA signals. To verify the 


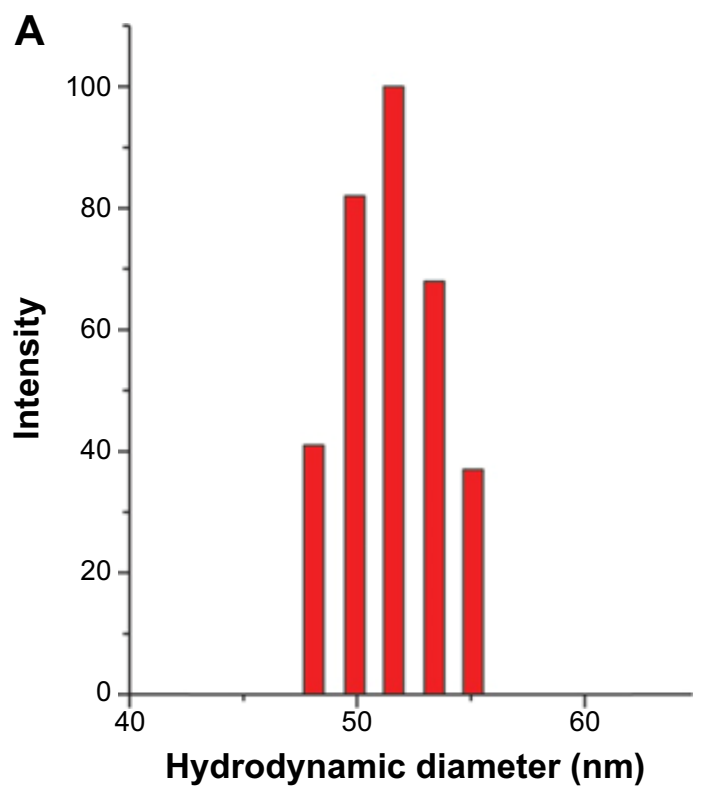

B
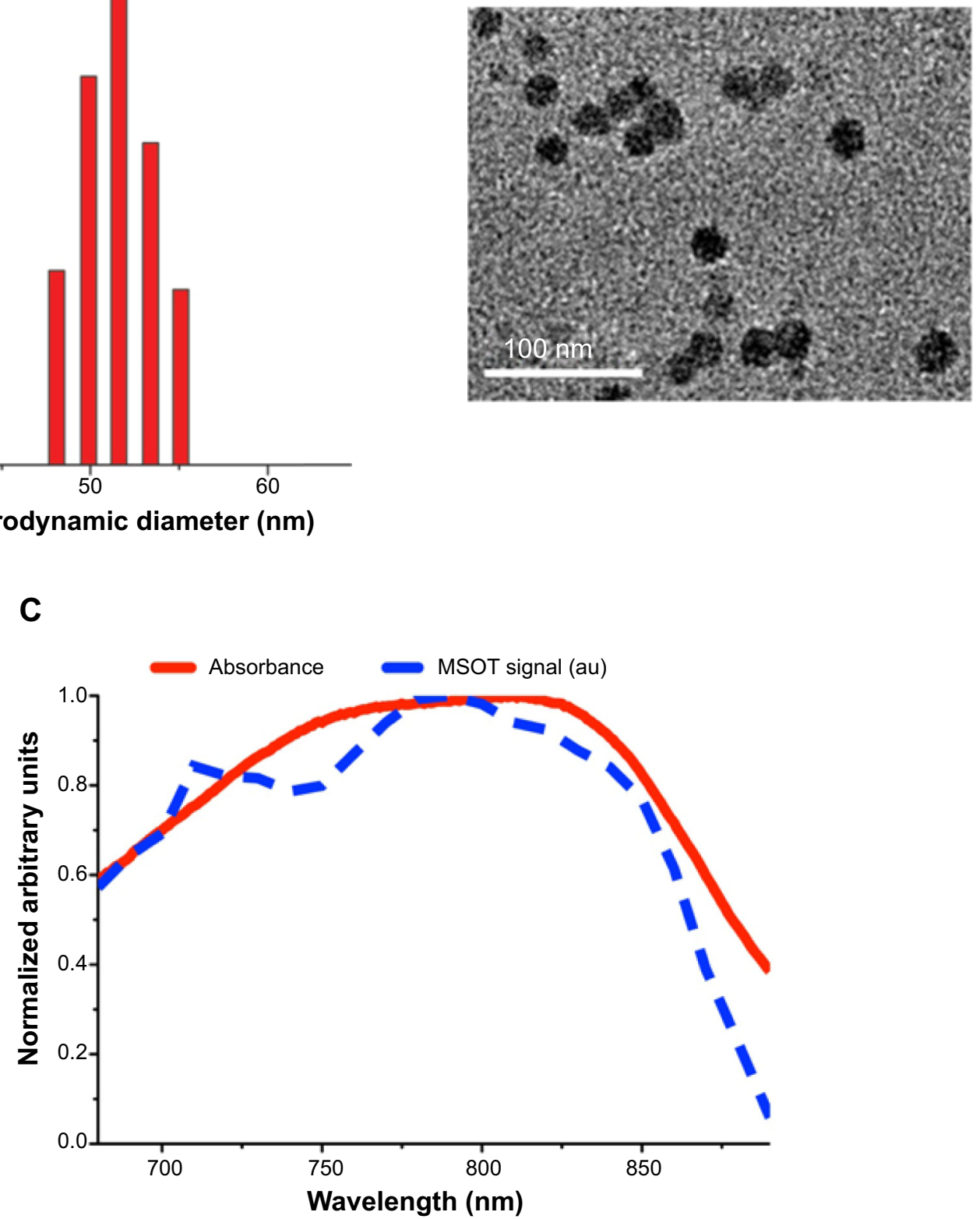

Figure 2 Characterization of folate-CP/CP dots.

Notes: (A) Particle size distribution of folate-CP dots studied by laser light scattering. (B) High-resolution transmission electron microscopy of CP dots. (C) Absorbance (red) and PA intensity (blue) as a function of wavelength. Similarity in trend between the optical absorption and PA intensity illustrates the strong correlation between the two. Abbreviations: au, arbitrary unit; CP, conjugated polymer; MSOT, multispectral optoacoustic tomography; PA, photoacoustic.

PA activity of this CP-based probe under controlled conditions, we performed phantom measurements for five different probe concentrations. After image reconstruction, results showed that the probe exhibits strong PA activity in phantoms, with a similar trend in waveform between absorbance and PA intensity as a function of wavelength, as shown in Figure 2C. This demonstrates a strong correlation between optical absorption and the PA effect, which validates our data, since the PA effect is mainly based on light absorption. In addition, after performing multispectral processing of the reconstructed PA signals to unmix the PA signal contribution from the probe, a linear relationship could be observed between the PA intensity and the concentration of the probe (Figure S2).

\section{In vitro cytotoxicity and target specificity assays}

As an essential issue in bioimaging both in vitro and in vivo, the cytotoxicity of folate-CP dots and $\mathrm{CP}$ dots was assessed 
in vitro on a FR+ve human mammary adenocarcinoma cell line, MCF-7. Figure 3A demonstrates the percentage of viable cells after exposing the cells to four different concentrations $(0.025,0.05,0.075$, and $0.1 \mathrm{mg} / \mathrm{mL})$ of folate-CP dots and CP dots for 24 hours. For concentrations up to $0.05 \mathrm{mg} / \mathrm{mL}$, cell viability was as high as $96 \%$ for folate-CP dots and $89 \%$ for $\mathrm{CP}$ dots. Therefore, a concentration of less than $0.05 \mathrm{mg} / \mathrm{mL}$ was chosen for in vitro and in vivo experiments. Moreover, for all the tested concentrations of up to $0.1 \mathrm{mg} / \mathrm{mL}$, the viability was more than $50 \%$ for both folate-CP dots and CP dots.

The specificity of folate-CP dots in targeting folate receptors on cells was tested in vitro on FR+ve MCF-7 cells at increasing concentrations of free folic acid. CP dots with no folate were used as a control. After exposure to $0.03 \mathrm{mg} / \mathrm{mL}$ of probes for 2 hours, the cells demonstrated an increased PA signal, with folate-CP dots-treated cells showing $\sim 5$-fold more PA signal than do CP dots-treated cells in the absence of free folic acid. However, with increasing free folic acid concentration, the PA activity decreased in folate-CP-dotstreated cells because of the competitive binding between folate-CP dots and free folic acid for folate receptors on MCF-7 cells, whereas the PA activity from CP dots-treated cells remained relatively the same (Figure $3 \mathrm{~B}$ ). This confirmed the specific targeting of folate ligands on folate-CP dots to the folate receptors on the cell surface, followed by internalization into cells through receptor-mediated endocytosis.

\section{In vivo molecular imaging of breast cancer}

Folate-CP/CP dots with a strong and broad absorption in the NIR region between 700 and $850 \mathrm{~nm}$ and strong PA activity

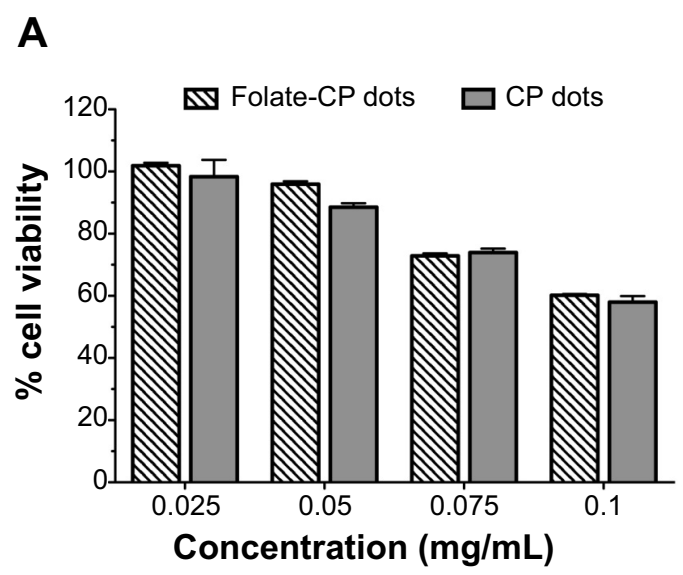

in phantoms and in vitro could be highly suitable for in vivo deep tissue PA imaging. In addition, its absorbance spectrum differs significantly from those of the major background tissue chromophores such as oxy- and deoxyhemoglobin (Figure S1), facilitating accurate multispectral unmixing of individual PA signals. To test the feasibility of applying folate-CP dots for noninvasive PA molecular imaging of folate receptors, they were injected intravenously into mice bearing $\mathrm{FR}+\mathrm{ve} \mathrm{MCF}-7$ breast cancer xenografts. CP dots with no folate functionalization were used as the control. PA signals were collected at five different excitation wavelengths - 700, 750, 810, 850, and $900 \mathrm{~nm}$ - based on the absorption maxima and minima of the probe, and oxy- and deoxyhemoglobin at 1, 3, 5, 24, 48, 72 , and 168 hours postadministration. To compensate for the attenuation of excitation light in deep tissues, the images were corrected using a wavelength-dependent exponential decay model, which assumes a uniform distribution of absorption and scattering properties, as well as constant tissue oxygenation. Subsequently, the images were spectrally decomposed to reveal the chromophore-specific contrast using known extinction spectra. Figure 4B-C are the MSOT maximum intensity projection images of the transverse and coronal abdomen sections of the tumor-bearing mice at 3 hours post folate-CP dots injection and Figure 4D-E are those injected with $\mathrm{CP}$ dots. PA signals at all other time points are provided in Figure S3. Figure $4 \mathrm{~F}$ is obtained from $\mathrm{ROI}$ analyses on single-slice images corresponding to the tumor site at various time points after probe injection. A strong PA signal could be detected as early as 1 hour in mice injected with folate-CP dots, which reached a maximum at 3 hours (Supplementary video) after which it gradually dropped, whereas the PA signal from the tumor in

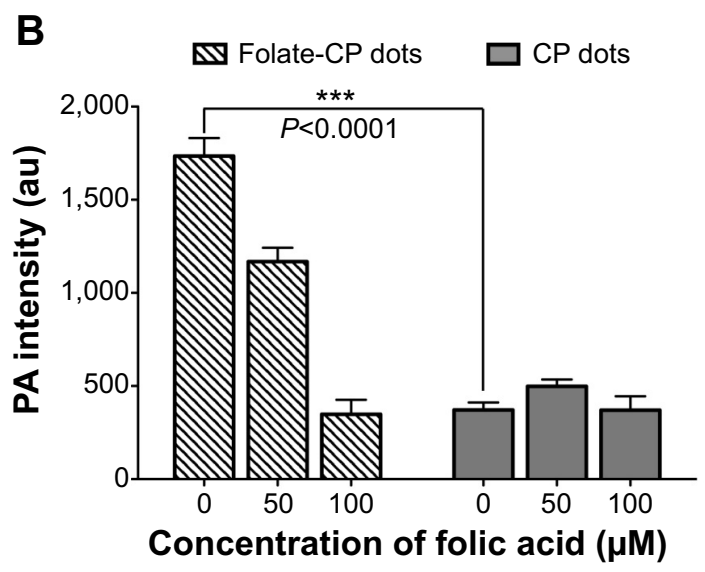

Figure 3 In vitro cytotoxicity and target specificity assays.

Notes: (A) In vitro cell viability after exposure to folate-CP dots and CP dots at various concentrations. (B) Specificity of folate-CP dots in targeting folate receptor-positive MCF-7 cells. PA signal from cells treated with folate-CP dots was $\sim 5$-fold higher than that from cells treated with CP dots after 2 hours of treatment in the absence of free folic acid. Data are represented as mean \pm SEM after statistical analysis using unpaired $t$-test; $P<0.1 ; * * * P<0.000$ I.

Abbreviations: au, arbitrary unit; $\mathrm{CP}$, conjugated polymer; PA, photoacoustic; SEM, standard error of the mean. 

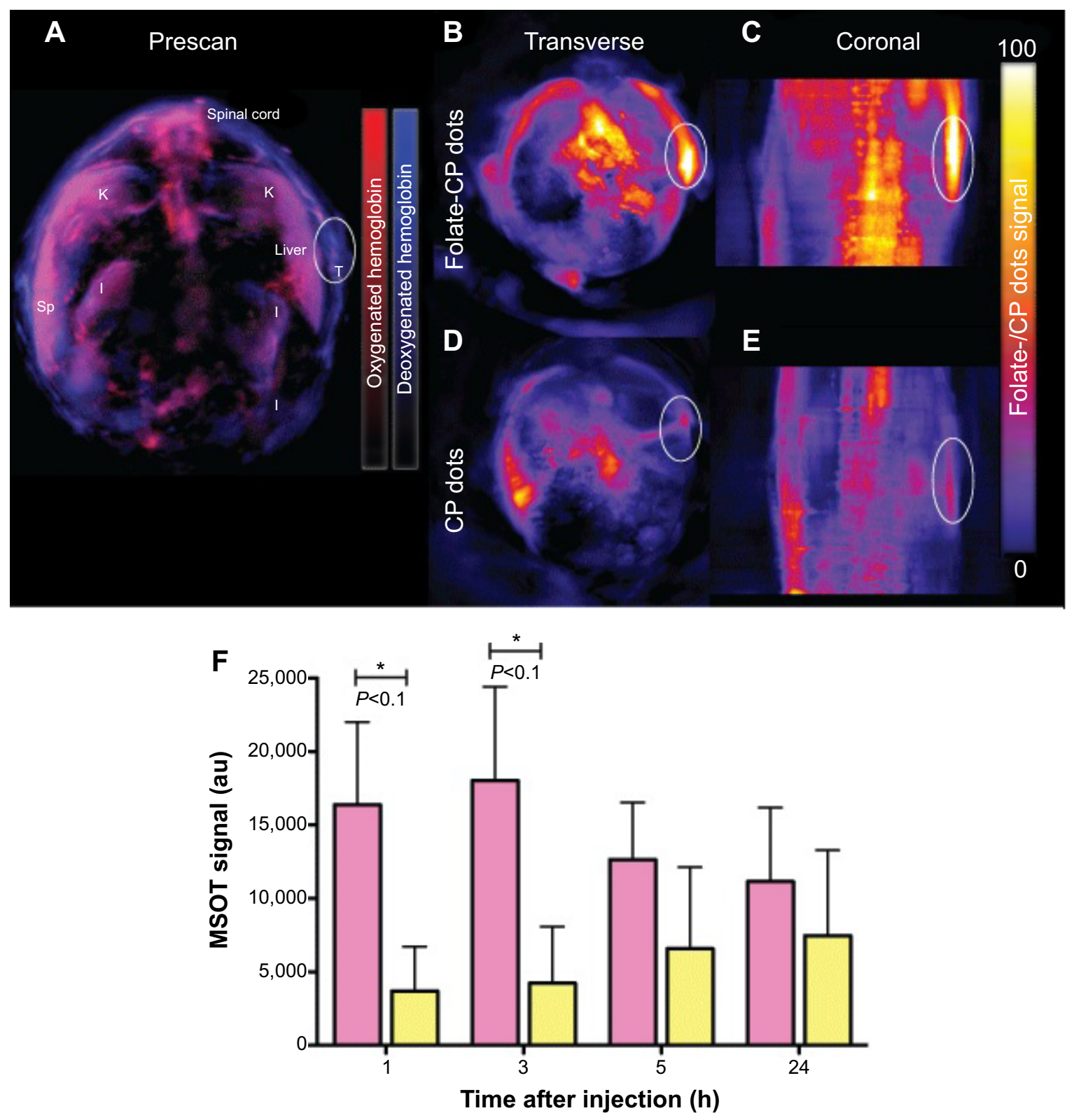

Folate-CP dots $\square$ CP dots

Figure 4 Molecular photoacoustic imaging of breast cancer.

Notes: MSOT MIP images of cross-sectional abdomen before systemic administration of the probe (A), at 3 hours after systemic administration of folate-CP dots (B, transverse view and $\mathbf{C}$, coronal view) and CP dots (D, transverse view and $\mathbf{E}$, coronal view). White ellipse indicates the location of the tumor. (F) Quantification of PA signal by ROI analysis at the tumor site after systemic administration of folate-CP-CP dots in FR+ve MCF-7 breast cancer tumor model ( $\mathrm{n}=3$ ). Data are represented as mean \pm SEM after statistical analysis using unpaired $t$-test; $P<0.1 ; * P<0.1$.

Abbreviations: au, arbitrary unit; $\mathrm{CP}$, conjugated polymer; FR+ve, folate receptor-positive; h, hour; MIP, maximum intensity projection; MSOT, multispectral optoacoustic tomography; PA, photoacoustic; ROI, region of interest; SEM, standard error of the mean; Sp, spleen; I, intestine; K, kidneys; T, tumor.

mice injected with CP dots was not as strong but gradually increased over time. This implies faster tumor localization of the folate-CP dots than that of CP dots. Since the EPR effect requires 6-24 hours, ${ }^{31,32}$ the accumulation of folate-CP dots at the tumor site during the first few hours after injection could be attributed to the active folate-mediated targeting instead of the EPR effect. Furthermore, the PA signal was more pronounced
( 4 times) in the tumor of folate-CP dots-treated mice than in the tumor of CP dots-treated mice up to 3 hours postinjection after which tumor PA signal from CP dots-treated mice increased (Figure 4F). These results demonstrate the feasibility of using folate-CP dots for molecular imaging of FR+ve breast cancer and delineating the tumor margin between 1 and 3 hours postinjection. 
A

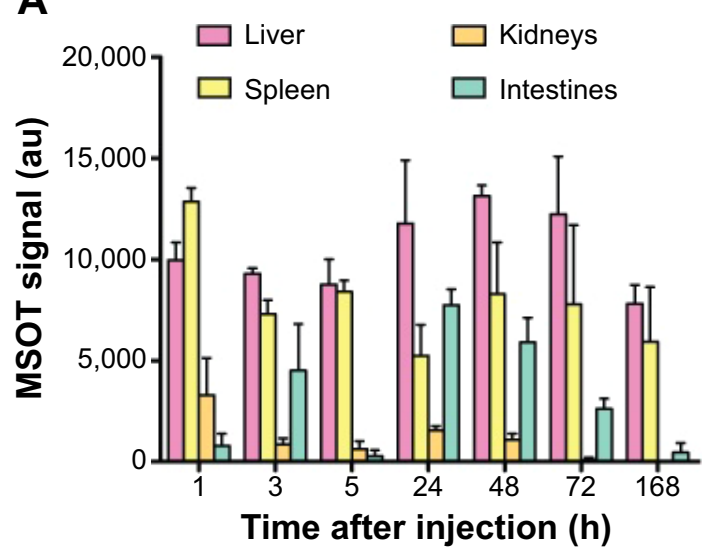

B

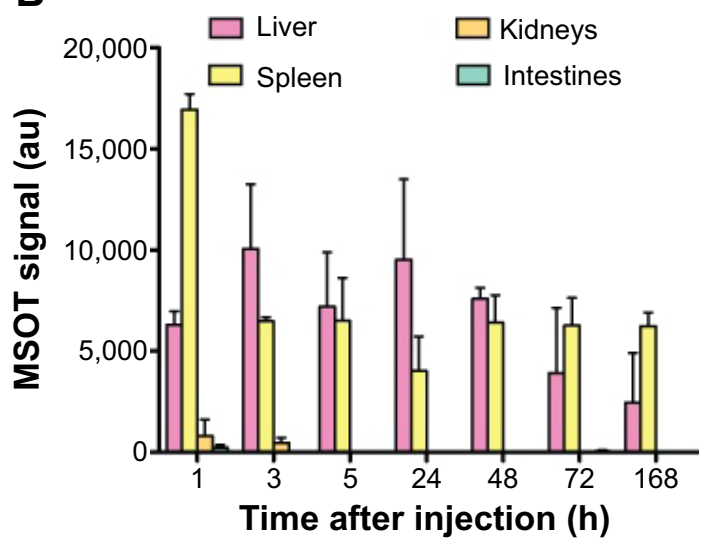

Figure 5 Semiquantitative biodistribution and pharmacokinetics.

Notes: Folate-CP dots (A) and CP dots (B) at various time points up to a week in liver, spleen, kidneys, and intestines of FR+ve MCF-7 breast cancer tumor model ( $\mathrm{n}=3$ ). Data are represented as mean \pm SEM after statistical analysis using unpaired $t$-test; $P<0$.I.

Abbreviations: au, arbitrary unit; FR+ve, folate receptor-positive; h, hours; MSOT, multispectral optoacoustic tomography; SEM, standard error of the mean.

\section{Semiquantitative biodistribution and clearance of folate-CP/CP dots}

Apart from monitoring the accumulation of contrast agents in tumor, MSOT, being a whole-body tomographic imaging system, can also provide semiquantitative information on the biodistribution of compounds in the body which can aid in the elucidation of clearance pathways and choosing of optimal dosing strategies. ${ }^{33}$ From the PA signal collected from the abdomen of the mice, maximum intensity projection images were obtained (Figure 4B-E) and ROI analyses were performed on individual organs such as liver, spleen, kidney, and intestines. Figure 5A and B illustrate that the PA signal from the probe could be detected in the liver and spleen immediately after the injection of both folate-CP dots and $\mathrm{CP}$ dots and for up to a week. PA signals in kidneys and intestines were barely detectable in $\mathrm{CP}$ dots-injected mice, whereas there was a considerable amount of signal in the kidneys and intestines of folate-CP-dots-injected mice. This difference could be attributed to the expression of folate receptors in the kidneys and intestines. ${ }^{34,35}$ This undesirable accumulation in the intestines and kidneys can be reduced by predosing the animals with folic acid or folic acid analogs such as pemetrexed while still retaining the desired $\mathrm{CP}$ dots accumulation in the tumor region. ${ }^{36}$ Moreover, the difference between the uptake in the kidneys or intestines in folate-CP dots-treated and CP dots-treated mice was not statistically significant $(P>0.1)$, and the tumor-to-kidney ratio was high, suggesting that the injected dose of folate-CP/CP dots may not be an overload on the kidneys. The strong PA signal in the liver and spleen suggests that the clearance of folate- $\mathrm{CP} / \mathrm{CP}$ dots could be through the hepatobiliary system. This is also in agreement with the general notion that nanoparticles of $\sim 60 \mathrm{~nm}$ size are cleared through the hepatobiliary system..$^{37,38}$

\section{Conclusion}

Herein, we have developed the first active-targeting CP-based probe with strong PA activity for in vivo noninvasive molecular imaging of breast cancer to the best of our knowledge. In particular, we verified the high PA signal of folate-CP dots in phantom models, as well as its application as a noninvasive PA contrast agent for molecular imaging of breast cancer in mice xenograft models in vivo. Folate-CP dots localized in the tumor site as fast as 1 hour postinjection to give a $\sim 4$-fold enhanced PA signal compared to $\mathrm{CP}$ dots, thus providing a better delineation from the surrounding tissue. Future studies will be carefully planned to investigate the possibility of translation for clinical use.

\section{Acknowledgments}

This work was supported by Singapore Bioimaging Consortium and Institute of Materials Research Engineering, Agency for Science, Technology, and Research, Singapore, and funded by Joint Council Office (Project Number: 1231BEG042), Agency for Science, Technology and Research, Singapore.

\section{Author contributions}

GB and CJHH contributed equally to the work in writing the main manuscript, performing experiments, and preparing figures. KL prepared the nanoparticles and characterized them for size. WD assisted in image postprocessing. USD and CLW assisted in in vivo experiments. VN, BL, and MO 
oversaw and managed the research project and directions. All authors contributed toward data analysis, drafting and revising the paper and agree to be accountable for all aspects of the work.

\section{Disclosure}

The authors report no conflicts of interest in this work.

\section{References}

1. Wang LV. Multiscale photoacoustic microscopy and computed tomography. Nat Photonics. 2009;3(9):503-509.

2. Ntziachristos V, Razansky D. Molecular imaging by means of multispectral optoacoustic tomography (MSOT). Chem Rev. 2010;110: 2783-2794.

3. Wang LV, Hu S. Photoacoustic tomography: in vivo imaging from organelles to organs. Science. 2012;335:1458-1462.

4. Ku, G, Wang X, Xie X, Stoica G, Wang LV. Imaging of tumor angiogenesis in rat brains in vivo by photoacoustic tomography. Appl Opt. 2005;44(5):770-775.

5. Stein EW, Maslov K, Wang LV. Noninvasive, in vivo imaging of bloodoxygenation dynamics within the mouse brain using photoacoustic microscopy. J Biomed Opt. 2009;14(2):020502.

6. Burton NC, Patel M, Morscher S, et al. Multispectral optoacoustic tomography (MSOT) of the brain and glioblastoma characterization. Neuroimage. 2013;65:522-528.

7. Mallidi S, Luke GP, Emelianov S. Photoacoustic imaging in cancer detection, diagnosis, and treatment guidance. Trends Biotechnol. 2011; 29(5):213-221.

8. Li ML, Wang JC, Schwartz JA, Gill-Sharp KL, Stoica G, Wang LV. In vivo photoacoustic microscopy of nanoshell extravasation from solid tumor vasculature. J Biomed Opt. 2009;14(1):010507.

9. Taruttis A, Herzog E, Razansky D, Ntziachristos V. Real-time imaging of cardiovascular dynamics and circulating gold nanorods with multispectral optoacoustictomography. Opt Express. 2010;18(19): 19592-19602.

10. Taruttis A, Wildgruber M, Kosanke K, et al. Multispectral optoacoustic tomography of myocardial infarction. Photoacoustics. 2013;1:3-8.

11. Stritzker J, Kirscher L, Scadeng M, et al. Vaccinia virus-mediated melanin production allows MR and optoacoustic deep tissue imaging and laser-induced thermotherapy of cancer. Proc Natl Acad Sci. 2013 110(9):3316-3320.

12. Kim C, Cho EC, Chen J, et al. In vivo molecular photoacoustic tomography of melanomas targeted by bioconjugated gold nanocages. ACS Nano. 2010;4(8):4559-4564.

13. Jokerst JV, Thangaraj M, Kempen PJ, Sinclair R, Gambhir SS. Photoacoustic imaging of mesenchymal stem cells in living mice via silica-coated gold nanorods. ACS Nano. 2012;6(7):5920-5930.

14. Jokerst JV, Cole AJ, van de Sompeland D, Gambhir SS. Gold nanorods for ovarian cancer detection with photoacoustic imaging and resection guidance via Raman imaging in living mice. ACS Nano. 2012;6(11): 10366-10377.

15. Homan KA, Souza M, Truby R, et al. Silver nanoplate contrast agents for in vivo molecular photoacoustic imaging. ACS Nano. 2012;6(1):641-650.

16. de la Zerda A, Bodapati S, Teed R, et al. Family of enhanced photoacoustic imaging agents for high-sensitivity and multiplexing studies in living mice. ACS Nano. 2012;6(6):4694-4701.

17. Buehler A, Herzog E, Ale A, Smith BD, Ntziachristos V, Razansky, D. High resolution tumor targeting in living mice by means of multispectral optoacoustic tomography. EJNIMMI Res. 2012;2:14.

18. Taruttis A, Morscher S, Burton NC, Razansky D, Ntziachristos V. Fast multispectral optoacoustic tomography (MSOT) for dynamic imaging of pharmacokinetics and biodistribution in multiple organs. PLOS ONE. 2012;7(1):e30491.
19. Herzog E, Taruttis A, Beziere N, Lutich AA, Razansky D, Ntziachristos V. Optical imaging of cancer heterogeneity with multispectral optoacoustic tomography. Radiology. 2012;263(2):461-468.

20. Li J, Liu J, Wei CW, Liu B, O’Donnell M, Gao X. Emerging applications of conjugated polymers in molecular imaging. Phys Chem Chem Phys. 2013;15:17006-17015.

21. Li K, Zhan R, Feng SS, Liu B. Conjugated polymer loaded nanospheres with surface functionalization for simultaneous discrimination of different live cancer cells under single wavelength excitation. Anal Chem. 2011;83:2125-2132.

22. Li K, Liu B. Polymer encapsulated conjugated polymer nanoparticles for fluorescence bioimaging. J Mater Chem. 2012;22:1257-1264.

23. Geng J, Li K, Pu KY, Ding D, Liu B. Conjugated polymer and gold nanoparticle co-loaded PLGA nanocomposites with eccentric internal nanostructure for dual-modal targeted cellular imaging. Small. 2012; 8(15):2421-2429.

24. Li K, Ding D, Huo D, et al. Conjugated polymer based nanoparticles as dual modal probes for targeted in vivo fluorescence and magnetic resonance imaging. Adv Funct Mater. 2012;22:3107-3115.

25. Zha Z, Deng Z, Li Y, et al. Biocompatible polypyrrole nanoparticles as a novel organic photoacoustic contrast agent for deep tissue imaging. Nanoscale. 2013;5:4462-4467.

26. Liu J, Geng J, Liao LD, Thakor N, Gaod X, Liu B. Conjugated polymer nanoparticles for photoacoustic vascular imaging. Polym Chem. 2014;5: 2854-2862.

27. Buehler A, Herzog E, Razansky D, Ntziachristos V. Video rate optoacoustic tomography of mouse kidney perfusion. Opt Lett. 2010;35: 2475-2477.

28. Rosenthal A, Razansky D, Ntziachristos V. Fast semi-analytical modelbased acoustic inversion for quantitative optoacoustic tomography. IEEE Trans Med Imag. 2010;29:1275-1285.

29. Li K, Jiang YH, Ding D, et al. Folic acid-functionalized two-photon absorbing nanoparticles for targeted MCF-7 cancer cell imaging. Chem Comm. 2011;47:7323-7325.

30. Li K, Qin W, Ding D, et al. Photostable fluorescent organic dots with aggregation-induced emission (AIE dots) for noninvasive long-term cell tracing. Sci Rep. 2013;3:1150.

31. Maeda $H$. The enhanced permeability and retention (EPR) effect in tumor vasculature: the key role of tumor-selective macromolecular drug targeting. Adv. Enzym Regul. 2001;41:189-207.

32. Maeda H, Nakamura H, Fang J. The EPR effect for macromolecular drug delivery to solid tumors: Improvement of tumor uptake, lowering of systemic toxicity, and distinct tumor imaging in vivo. Adv Drug Deliv Rev. 2013;65(1):71-79.

33. Ho CJH, Balasundaram G, Driessen W, et al. Multifunctional photosensitizer-based contrast agents for photoacoustic imaging. Sci Rep. 2014;4:5342.

34. Parker N, Turk MJ, Westrick E, Lewis JD, Low PS, Leamon CP. Folate receptor expression in carcinomas and normal tissues determined by a quantitative radioligand binding assay. Anal Biochem. 2005:338(2):284-293.

35. Luo Z, Chen Y, Chen S, et al. Comparison of inhibitors of superoxide generation in vascular smooth muscle cells. Br J Pharmacol. 2009;157(6):935-943.

36. Müller C, Schibli R. Folic acid conjugates for nuclear imaging of folate receptor-positive cancer. $J$ Nucl Med. 2011;52(1):1-4.

37. Moghimi SM, Hunter AC, Murray JC. Long-circulating and target-specific nanoparticles: theory to practice. Pharmacol Rev 2001;53(2):283-318.

38. Moghimi SM, Hunter AC, Murray JC. Nanomedicine: current status and future prospects. FASEB J. 2005;19(3):311-330. 


\section{Supplementary materials} Calculation of folate density on folate-CP dot surface

Freeze-drying of the folate-CP dot stock solution $(9 \mathrm{~mL})$ yielded $2.5 \mathrm{mg}$ of powder. As the folate-CP dots are stable in water, the density of the dot suspension could be estimated as $\sim 1 \mathrm{~g} / \mathrm{cm}^{3}$. As the average size is $\sim 51.5 \mathrm{~nm}$, the concentration of the folate-CP dots in stock can be calculated from the following equation:

Total number of folate-CP dots in $9 \mathrm{~mL}$ of suspension

$=\frac{\text { Total volume of folate-CP dots }}{\text { Average volume of each dot }}$

$$
=\frac{\frac{2.5 \times 10^{-3} \mathrm{~g}}{1 \mathrm{~g} / \mathrm{mL}}}{\frac{4}{3} \pi \times\left(25.75 \times 10^{-7}\right)^{3} \mathrm{~mL}}=3.4 \times 10^{13}
$$

Finally, the concentration of folate-CP dots in stock solution was calculated as follows:

$$
[\text { Folate-CP dots }]=\frac{\frac{3.4 \times 10^{13}}{6.02 \times 10^{23} \mathrm{~mol}^{-1}}}{9 \times 10^{-3} \mathrm{~L}}=6.2 \mathrm{nM}
$$

The feeding amounts of DSPE-PEG-folate, DSPE-PEG, and $\mathrm{CP}$ during folate-CP dots preparation is $1 \mathrm{mg}$ for each. Assuming the sample loss during folate-CP dots synthesis is consistent with the feeding ratio, the amount of folate in the final obtained dots suspension can be calculated as follows:

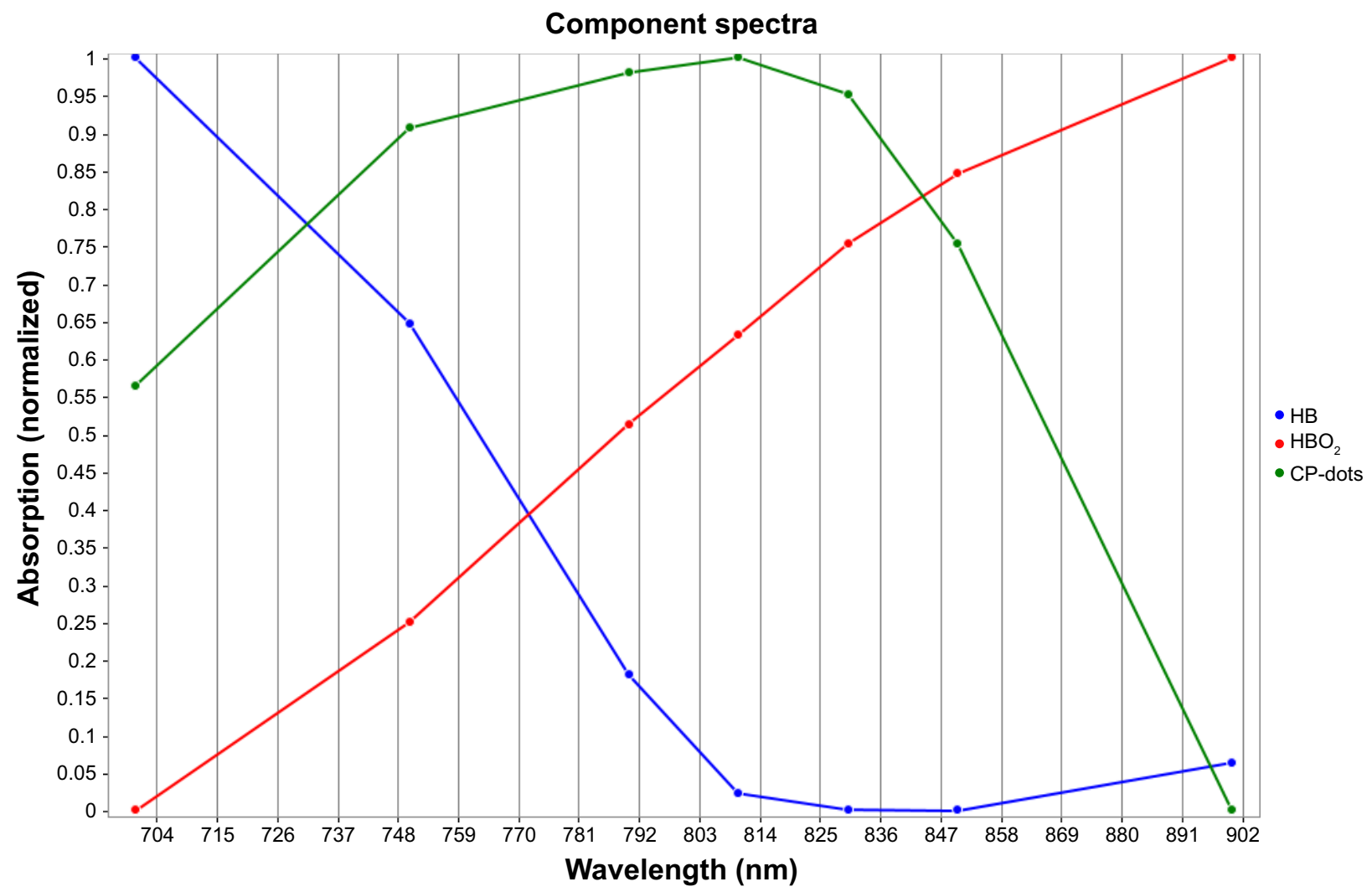

Figure SI Normalized absorbance spectra of deoxyhemoglobin $(\mathrm{HB})$, oxyhemoglobin $\left(\mathrm{HBO}_{2}\right)$ and conjugated polymer $(\mathrm{CP})$ dots.

Notes: This figure shows that the absorbance spectrum of CP dots differs significantly from those of the major background tissue chromophores such as oxy- and deoxyhemoglobin. 


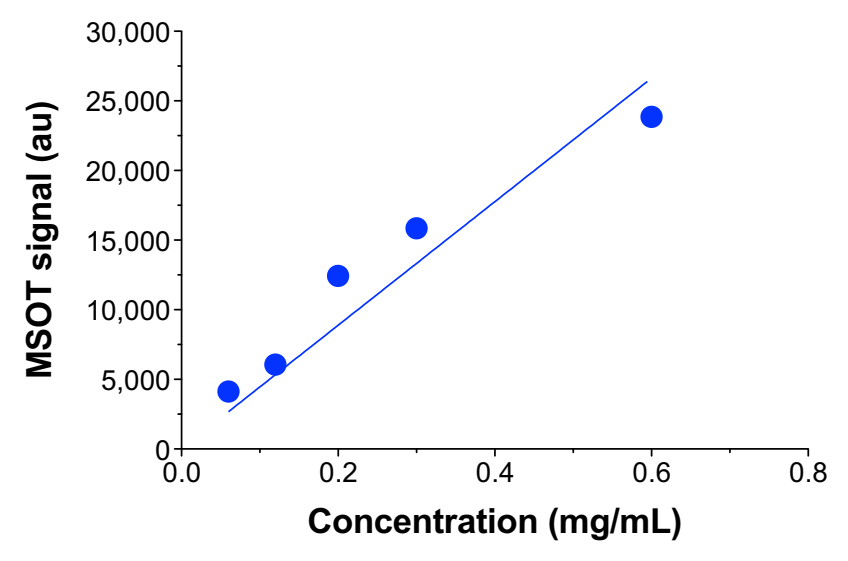

Figure S2 Linear relationship between the PA intensity and the concentration of the folate-CP dots.

Abbreviations: au, arbitrary unit; $\mathrm{CP}$, conjugated polymer; MSOT, multispectral optoacoustic tomography; PA, photoacoustic.

$$
[\text { Folate }]=\frac{\frac{1 \times \frac{2.5}{3}}{3230} \times 10^{-3} \mathrm{~mol}}{9 \times 10^{-3} \mathrm{~L}}=2.8 \times 10^{-5} \mathrm{M}
$$

As a result, the number of folate molecules on each dot can be obtained:

$$
[\text { Folate } / \mathrm{dot}]=\frac{2.8 \times 10^{-5} \mathrm{M}}{6.2 \times 10^{-9} \mathrm{M}}=4516
$$

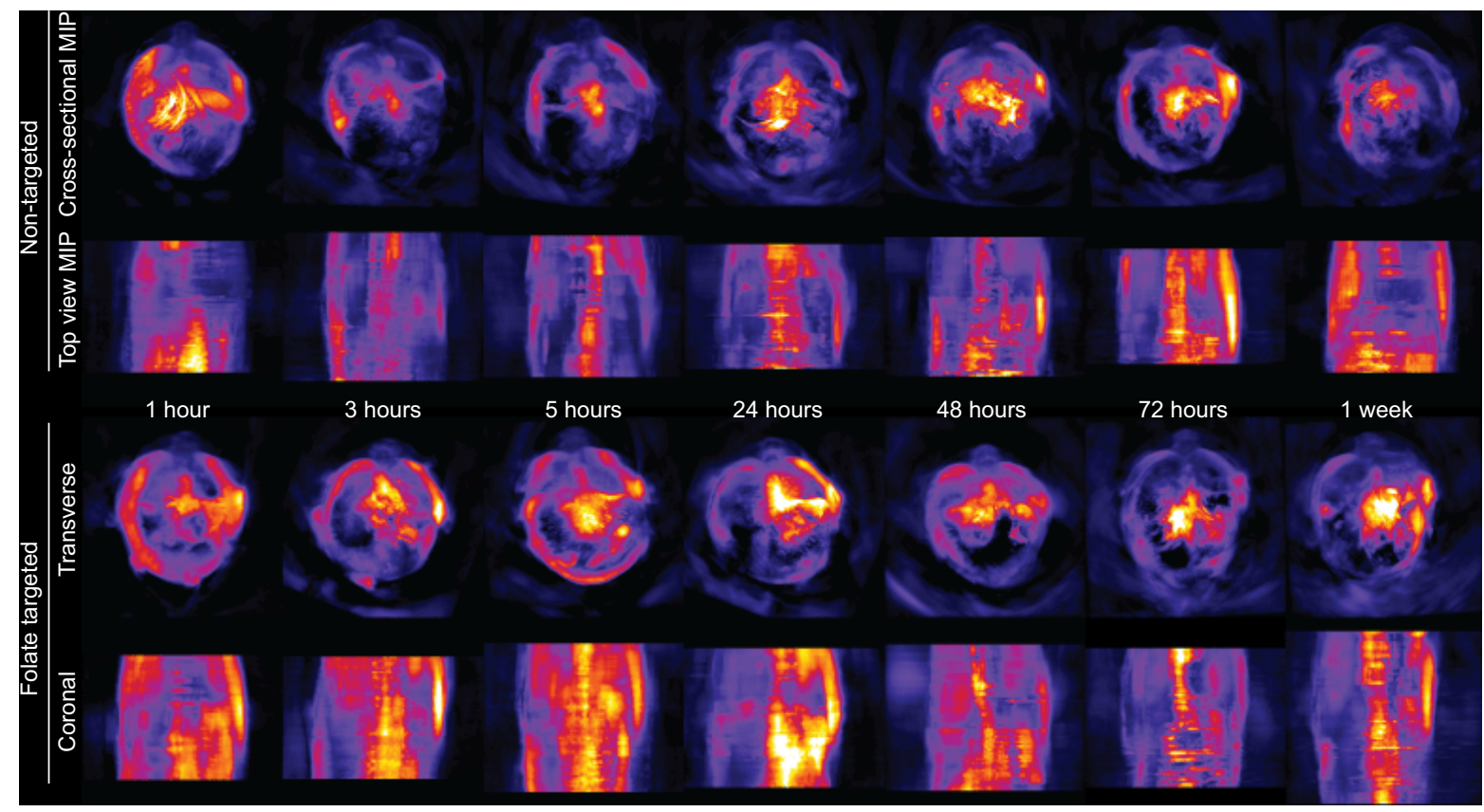

Figure S3 MSOT maximum intensity projection (MIP) images of cross-sectional and top views of mouse, showing the probe biodistribution at various time points after injection.

Abbreviation: MSOT, multispectral optoacoustic tomography.

Video S1 A video depicting the strong PA signal at the tumor site 3 hours after folate-CP dots injection in MCF-7 breast cancer xenograft model.

International Journal of Nanomedicine

\section{Publish your work in this journal}

The International Journal of Nanomedicine is an international, peerreviewed journal focusing on the application of nanotechnology in diagnostics, therapeutics, and drug delivery systems throughout the biomedical field. This journal is indexed on PubMed Central, MedLine, CAS, SciSearch $\AA$, Current Contents ${ }^{\circledR} /$ Clinical Medicine,

\section{Dovepress}

Journal Citation Reports/Science Edition, EMBase, Scopus and the Elsevier Bibliographic databases. The manuscript management system is completely online and includes a very quick and fair peer-review system, which is all easy to use. Visit http://www.dovepress.com/ testimonials.php to read real quotes from published authors.

\footnotetext{
Submit your manuscript here: http://www.dovepress.com/international-journal-of-nanomedicine-journal
} 